\title{
Polypharmacy and adverse drug reactions in Japanese elderly taking antihypertensives: a retrospective database study
}

This article was published in the following Dove Press journal:

Drug, Healthcare and Patient Safety

22 June 2013

Number of times this article has been viewed

\author{
Izumi Sato' \\ Manabu Akazawa ${ }^{2}$ \\ 'Department of Epidemiology \\ and Statistics, Graduate School of \\ Medicine, The University of Tokyo \\ Tokyo, Japan; ${ }^{2}$ Department of Public \\ Health and Epidemiology, Meiji \\ Pharmaceutical University, Tokyo, \\ Japan
}

Background: The concomitant use of multiple medications by elderly patients with hypertension is a relatively common and growing phenomenon in Japan. This has been attributed to several factors, including treatment guidelines recommending prescription of multiple medications and a continuing increase in the elderly population with multiple comorbidities.

Objective: This study was aimed at investigating the association between polypharmacy, defined as the concomitant use of five or more medications, and risk of adverse drug reaction (ADR) in elderly Japanese hypertensive patients to examine the hypothesis that risk of ADR increases with the administration of an increasing number of co-medications.

Methods: Using a retrospective cohort design, the data regarding all hypertensive patients aged 65 years or older were extracted from the Risk/Benefit Assessment of Drugs - Analysis and Response Council antihypertensive medication database. The data were reviewed for classification of patients into one of three groups according to drug use at the initiation of therapy - a monotherapy group composed of patients who had taken the investigated drug only, a comedication group composed of patients who had taken the investigated drug and a maximum of three other medications, and a polypharmacy group composed of patients who had taken the investigated drug and four or more other medications - and determination of the number of ADR events experienced. Estimated rate ratios (RRs) and 95\% confidence intervals (CIs) were calculated using a Poisson regression model adjusted for drug category and patient age and sex. Various sensitivity analyses were performed to confirm the robustness of the study findings.

Results: Of 61,661 elderly Japanese patients (men, 41.8\%; 75 years or older, 35.1\%) registered in the database, 2491 patients (4.0\%) experienced a total of 3144 ADR events during the study period. The rate of ADR per 10,000 person-days was 2.0 for the monotherapy group, 5.1 for the co-medication group, and 8.6 for the polypharmacy group. After adjusting for age, sex, and initial antihypertensive therapy, the RR was estimated at 2.4 (95\% CI, 2.2-2.6) for the co-medication group and 4.3 (95\% CI, 3.8-4.8) for the polypharmacy group, when compared with the monotherapy group.

Conclusion: The use of polypharmacy increases the risk of ADR among elderly Japanese patients with hypertension, calling for regular medication review to eliminate the administration of unnecessary co-medications.

Keywords: adverse drug reaction, antihypertensive, elderly, pharmacoepidemiology, polypharmacy

\section{Introduction}

Incorrect use of medications, a phenomenon estimated to occur with more than half of all medications currently prescribed, can have dire consequences for patients and health care in general. ${ }^{1}$ Such consequences, which include development of antimicrobial 
resistance, adverse drug reaction (ADR) events, erroneous prescription of medications, erosion of patient confidence, and waste of resources, have often been attributed to polypharmacy, commonly defined as either the use of multiple medications or the use of a medication without rational indication for its use. ${ }^{2,3}$ Cases of polypharmacy are generally described as either minor, defined as concurrent use of two to four medications, or major, defined as concurrent use of five or more medications, to describe the extent of concurrent medication use, but not the appropriateness of the medications prescribed. ${ }^{4}$

Previous studies in elderly populations have not only confirmed that the consequences of polypharmacy include ADR events, drug interactions, and prescription of inappropriate medications, but also observed that ADR events due to polypharmacy may be misdiagnosed as the onset of new symptoms or comorbidities. ${ }^{2,5-10}$ This latter phenomenon may in turn lead to increases in the extent of polypharmacy as well as increased medical costs and ultimately, medication errors due to the complexity of the medication regimens. ${ }^{10}$ Other studies have found that polypharmacy may lead to poor adherence to medication regimens, unsatisfactory therapeutic outcomes, and lowered quality of life..$^{5,8}$

One of the most common adult diseases worldwide, hypertension has been reported to affect approximately 6.4 million $(60 \%)$ people of the Japanese population over the age of 65 years. ${ }^{11}$ As hypertensive patients are more likely to have comorbid conditions and are required to maintain stringent control of blood pressure, elderly Japanese hypertensive patients are more likely to use multiple medications. ${ }^{12-19}$ Based on the hypothesis that administration of relatively low doses of multiple medications results in higher tolerability than administration of relatively high doses of one medication, current clinical guidelines for the treatment of hypertension recommend combination therapies if needed, thus increasing the potential for polypharmacy in hypertensive patients. ${ }^{15,16}$

Despite the potentially dire consequences of polypharmacy, few studies examining the association between polypharmacy and ADR risk in Japanese elderly patients have been conducted. One study of 1289 elderly inpatients in five university hospitals found that among the $9.2 \%$ of inpatients experiencing ADR events, the mean number of drugs taken on admission was 5.7, compared to a mean of 5.0 by those who were not experiencing ADR events. ${ }^{20}$ Another study of 517 elderly patients with dementia treated at a single hospital found that among the $12.7 \%$ of inpatients experiencing ADR events, $15 \%$ were taking five or more medications. ${ }^{21}$
However, these studies considered neither the duration of medication use, nor the presence of comorbidities, and included a limited number of medical institutions and clinics, decreasing the ability to generalize the findings to the general Japanese elderly population.

Under the Japanese post-marketing surveillance (PMS) system, data regarding the safety and effectiveness of a drug after it has been introduced into the market are mainly collected through the spontaneous reporting system and drug use investigations (DUIs). ${ }^{22-24}$ After a new drug has been marketed for 4 to 10 years, pharmaceutical companies are required to submit the results of PMS studies to conduct what is referred to as a "re-examination" of the drug. To ensure the quality of PMS data utilized for the re-examination, data collection must adhere to a regulation originally entitled "Good Post-Marketing Surveillance Practice" (GPMSP) before being subsequently revised in two regulations entitled "Good Post-Marketing Study Practice" (GPSP) and "Good Vigilance Practice" (GVP) in 2004. ${ }^{22-24}$ Under these regulations, doctors who take part in a DUI must register patients newly prescribed the relevant drug during daily medical practice. Usually continuous surveillance and a central registration system are selected to minimize selection bias. Doctors must then record data regarding patient demographic characteristics, medical history, medication use, and disease conditions using a case report form (CRF) prepared by a pharmaceutical company. During a predetermined period for each drug, usually 3 to 6 months, doctors must monitor patients prospectively, recording the results of any laboratory tests conducted and the development of any ADR events. The pharmaceutical company then collects a target number of CRFs, typically 3000 to 10,000 , and summarizes the safety and effectiveness data for the re-examination requirement.

After the re-examination has been conducted by a pharmaceutical company, the Risk/Benefit Assessment of DrugsAnalysis and Response (RAD-AR) Council of Japan collects the DUI data examined by the company and pools them with data collected from other companies to construct databases. ${ }^{25}$ One current database constructed by the RAD-AR Council is the antihypertensive medication database, which contains data collected from 21 PMS studies conducted by 17 pharmaceutical companies between 1981 and $1999 .{ }^{26}$ Defined as the investigated drugs in this study, the antihypertensive medications are categorized as calcium channel blockers (CCBs), angiotensin-converting enzyme inhibitors (ACEIs), betablockers (BBs), alpha-blockers (ABs), or diuretics. Because the data structure and quality varied among the PMS studies, the RAD-AR Council standardized the data contained in the 
database using the International Classification of Diseases, 9th Revision, Clinical Modification (ICD-9-CM) codes for comorbid conditions; ${ }^{27}$ Medical Dictionary for Regulatory Activities (MedDRA) codes for ADR events; ${ }^{28,29}$ and drug classification codes for concomitant medications; ${ }^{30}$ as well as confirmed the validity of the redefined data by comparing them with the original data and verified their internal consistency.

This database has been used in various pharmacoepidemiological studies in Japan, including studies of the risk of dry cough due to ACEI administration, ${ }^{31}$ the effect of concomitant use of CYP3A4 inhibitors and CCBs in terms of ADR incidence, ${ }^{32}$ and the effect of nonsteroidal anti-inflammatory drugs in hypertensive patients on antihypertensive therapy. ${ }^{33}$ The anonymous database includes separate files containing data regarding patient demographics, medical conditions, medications, and ADR events that can be linked for analyses using a non-identifiable personal number created by the RAD-AR Council.

The current study was aimed at providing safety information for physicians and pharmacists regarding the treatment of elderly hypertensive patients, especially who were exposed to multiple medications. To do so, it examined the association between polypharmacy and ADR risk in elderly Japanese patients using a design and data source that overcame the limitations of previous studies.

\section{Materials and methods Study design and data source}

Using a retrospective cohort design that complied with the administrative rules and regulations of the RAD-AR Council, data regarding approximately 143,000 hypertensive patients were extracted from the antihypertensive medication database, constructed using the PMS data. ${ }^{25,34}$ Need for study approval by an institutional review board was waived because only secondary data containing no personal identifiers were extracted and analyzed.

\section{Study subjects}

The data regarding all hypertensive patients recorded in the database were reviewed for identifying all patients aged 65 years or older. Any patients whose age or sex was not specified were excluded from further consideration. Patient use of the investigated drug and of concomitant medications, regardless of class, at the initiation of treatment was determined to classify patients into one of three drug use groups: the monotherapy group, defined as patients who had taken the investigated drug only; the co-medication group, defined as patients who had taken the investigated drug and a maximum of three other medications, including antihypertensive agents; and the polypharmacy group, defined as patients who had taken the investigated drug and four or more other medications.

\section{Variables}

To examine the extent to which the patients had experienced ADR events, the outcome of interest, data regarding the date, severity, and intervention required (ie, dosage reduction or discontinuation) were extracted regarding all ADR events that had occurred. Data regarding the duration (in days) from initiation of the investigated drug to the end of follow-up were used to define the study period. Other explanatory variables used in the analyses included the category of the investigated drug ( $\mathrm{CCB}, \mathrm{ACEI}, \mathrm{BB}, \mathrm{AB}$, or diuretic), the number of co-medications taken (1, 2 to 4 , or 5 or more), patient age (65 to 74 years versus 75 years or older), and patient sex (male versus female).

\section{Statistical analysis}

Subject characteristics were described in terms of their frequency. The crude risk of ADR was calculated by dividing the number of ADR events by person-days for each drug use group. Estimated rate ratios (RRs) and 95\% confidence intervals (CIs) were calculated using a Poisson regression model adjusted for drug category and patient age and sex.

Various sensitivity analyses were performed to confirm the robustness of the study findings. In the first analysis, only severe ADR events, defined as those that required any actions, such as dosage reduction or discontinuation, were included. In the second analysis, which focused on investigating the long-term effects of polypharmacy, only subjects who had taken the investigated drug for at least 90 days were included. In the third analysis, data regarding the symptoms of "coughing" (MedDRA code: 10011232) or "cough" (MedDRA code: 10011224), which are wellknown as ADR events for ACEIs, and thus more likely to be reported by doctors in PMS studies, were excluded to reduce potential reporting bias. In the final analysis, the fact that several patients had begun taking additional medications subsequent to initiation of the study was considered by classifying patients into three groups according to the maximum number of medications that they had taken at any point during the study period, and by such means, the extent of misclassification bias was determined.

All statistical analyses were performed using SAS statistical software, version 9.3 (SAS Institute Inc, Cary, 
NC, USA). All $P$-values less than 5\% were considered statistically significant.

\section{Results}

The process of selecting the study population from the antihypertensive medication database is displayed in Figure 1. Of 61,792 patients aged 65 years or older identified, 131 were excluded from the analysis: 112 patients were excluded because the date of medication initiation/end or date of experiencing an ADR event was not recorded; 19 patients were excluded because their sex was not specified. Among the remaining 61,661 patients, 38,888 (63.1\%) were classified into the monotherapy group, 19,335 (31.4\%) into the comedication group, and 3438 (5.6\%) into the polypharmacy group based on a review of their medication history. The median duration, 25 th percentile, and 75 th percentile of the follow-up period were 111,84 , and 178 days, respectively.

The characteristics of the study subjects are summarized in Table 1. ACEIs were the most frequently prescribed agents in all three groups, followed by CCBs and BBs. No significant differences in age and sex distribution among the groups were observed.

The risk of ADR according to drug group in persondays is summarized in Table 2. Further, 2491 (4.0\%) of the subjects experienced 3144 ADR events, an overall incidence of 3.3 events per 10,000 person-days, and the most frequently experienced ADR events were coughing or cough followed by dizziness. The rate of ADR per 10,000 person-days was 2.0 for the monotherapy group, 5.1 for the co-medication group, and 8.6 for the polypharmacy group. After adjusting for age, sex, and the investigated drug, the RRs for comedication and polypharmacy groups were estimated at 2.4 (95\% CI, 2.2-2.6) and 4.3 (95\% CI, 3.8-4.8), respectively, when compared to the monotherapy group.

The results of the sensitivity analysis are summarized in Table 3. As can be observed, when the analysis was restricted to events that required intervention, the risk of ADR increased for both the co-medication and polypharmacy groups compared with the monotherapy group. A similar trend was observed when subjects who had taken the investigated drug for fewer than 90 days were excluded, when symptom of coughing or cough was excluded as an ADR event, and when the maximum number of medications taken was used to classify the patients into drug groups.

\section{Discussion}

The primary finding in this investigation of the association between polypharmacy and risk of ADR in elderly Japanese

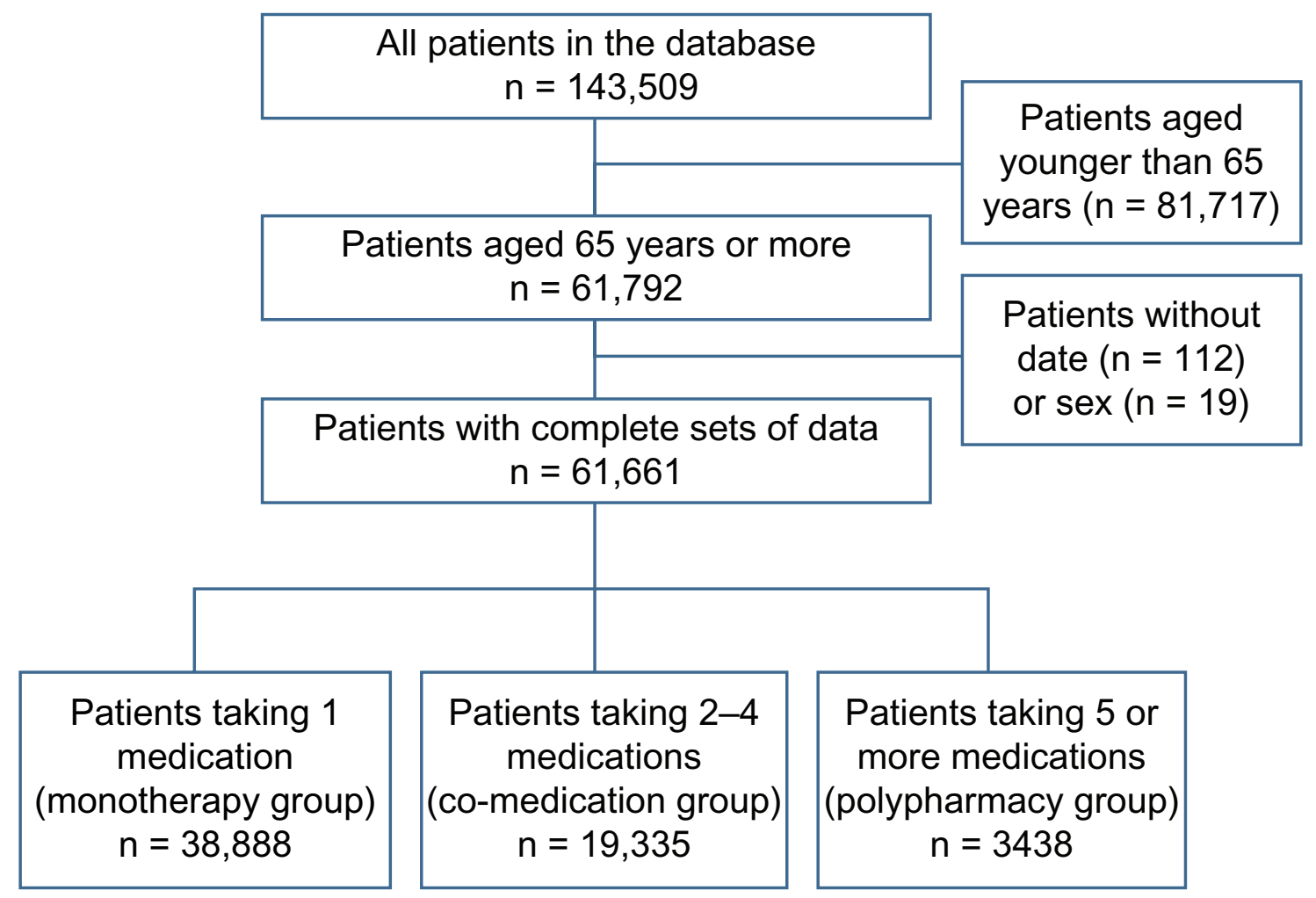

Figure I Flowchart of subject selection from the review of patient data in the Risk/Benefit Assessment of Drugs - Analysis and Response Council antihypertensive medication database. 
Table I Characteristics of study subjects

\begin{tabular}{|c|c|c|c|c|}
\hline & \multirow[t]{2}{*}{ All groups } & \multicolumn{3}{|l|}{ Drug use group } \\
\hline & & Monotherapy n (\%) & Co-medication (\%) & Polypharmacy (\%) \\
\hline Total & $61,661(100)$ & $38,888(63.1)$ & $19,335(31.4)$ & $3438(5.6)$ \\
\hline \multicolumn{5}{|c|}{ Age group (years) } \\
\hline $65-74$ & $39,989(64.9)$ & $25,956(66.7)$ & $12,069(62.4)$ & $1964(57.1)$ \\
\hline$\geq 75$ & $21,672(35.1)$ & I2,932 (33.3) & $7266(37.6)$ & 1474 (42.9) \\
\hline \multicolumn{5}{|l|}{ Sex } \\
\hline Male & $25,79 \mid(4 I .8)$ & I6,286 (4I.9) & $7987(4 \mid .3)$ & $1518(44.2)$ \\
\hline Female & $35,870(58.2)$ & $22,602(58.1)$ & I I,348 (58.7) & $1920(55.8)$ \\
\hline \multicolumn{5}{|c|}{ Anti-hypertensive drug used } \\
\hline $\mathrm{CCB}$ & $13,018(2||)$. & $5449(14.0)$ & $646 \mathrm{I}(33.4)$ & 1108 (32.2) \\
\hline ACEI & $24,77 I(40.2)$ & I2,747 (32.8) & $10,350(53.5)$ & $1674(48.7)$ \\
\hline BB & $16,11 \mid(26.1)$ & $13,720(35.3)$ & $1908(9.9)$ & $483(14.0)$ \\
\hline$A B$ & $4867(7.9)$ & $4078(10.5)$ & $616(3.2)$ & $173(5.0)$ \\
\hline Diuretic & $2894(4.7)$ & 2894 (7.4) & $0(0.0)$ & $0(0.0)$ \\
\hline
\end{tabular}

Note: Subjects were classified into three groups according to the initial number of medications taken.

Abbreviations: $A B$, alpha-blocker; $A C E I$, angiotensin-converting enzyme inhibitor; $B B$, beta-blocker; $C C B$, calcium channel blocker.

patients with hypertension was an increasing risk of ADR with an increasing number of medications taken after adjustment for patient characteristics. This finding was confirmed by the results of various sensitivity analyses and is consistent with previous studies that reported a positive association between polypharmacy and the risk of ADR. ${ }^{2,9,10}$

In Japan, so-called automated databases such as claims databases, medical records databases, and cancer registries databases have been developed for use in any type of research. Nevertheless, as only several large databases are currently available, data for the analyses conducted in this study were collected from the RAD-AR Council antihypertensive medication database, which contains the records of approximately 143,000 hypertensive patients. ${ }^{25}$ Pharmaceutical companies that release new drugs into the Japanese marketplace are required to conduct PMS studies to collect data regarding the safety and effectiveness of drugs in the course of medical practice that are of sufficiently high quality for use in the re-examinations. ${ }^{22-24}$ Even though the post-marketing studies may have possible limitations such as patient selection and reporting bias, in this study, a positive association was identified between ADR risk and number of co-medications.
In accordance with several previous studies, polypharmacy was defined as the use of five or more medications in this study. Such numerical definitions of polypharmacy often do not address the appropriateness of the medications, focusing solely on the extent of their concurrent use. ${ }^{2,3}$ The definition, however, is supported with the findings of two previous studies in Japan, which reported that patients who experience ADR events are likely to be taking five or more medications. ${ }^{20,21}$ Using the widely accepted definition of polypharmacy, greater risk of ADR was identified in the polypharmacy group than in the monotherapy or comedication groups in this study.

Further, in accordance with previous pharmacoepidemiology studies, a Poisson regression model was used to estimate the risk of ADR associated with each drug use group. A similar model was used by Ray et al in the evaluation of the risk of sudden cardiac death according to antipsychotic use (ie, current, former, or no use) $)^{35}$ and by McAfee et al to estimate incidence of drug-induced hospitalization or inhospital death among patients treated with statins. ${ }^{36}$ In the base case analysis, the number of medications being taken at baseline was determined for assigning the subjects into one of

Table 2 Number of person-days, number of ADR events, ADR rate, and estimated rate ratio

\begin{tabular}{|c|c|c|c|c|}
\hline & \multirow[t]{2}{*}{ All groups } & \multicolumn{3}{|c|}{ Drug use group } \\
\hline & & Monotherapy & Co-medication & Polypharmacy \\
\hline Number of person-days & $9,568,650$ & $6,074,669$ & $2,997,958$ & 496,023 \\
\hline Number of ADR events & 3144 & 1196 & 1519 & 429 \\
\hline ADR rate per 10,000 person-days & 3.3 & 2.0 & 5.1 & 8.6 \\
\hline Estimated rate ratio & - & 1.0 & 2.4 & 4.3 \\
\hline $95 \%$ confidence interval & - & - & $2.2-2.6$ & $3.8-4.8$ \\
\hline
\end{tabular}

Note: ADR rate ratios were estimated using a Poisson regression model adjusted for age, sex, and drug category.

Abbreviation: ADR, adverse drug reaction. 
Table 3 Results of sensitivity analyses

\begin{tabular}{|c|c|c|c|}
\hline & \multicolumn{3}{|l|}{ Drug use group } \\
\hline & Monotherapy & Co-medication & Polypharmacy \\
\hline \multicolumn{4}{|c|}{ Inclusion of severe ADR events only } \\
\hline Person-days of follow-up & $6,074,669$ & $2,997,958$ & 496,023 \\
\hline Number of ADR events & 763 & 930 & 249 \\
\hline ADR rate per 10,000 person-days & 1.3 & 3.1 & 5.0 \\
\hline Estimated rate ratio & I & 2.6 & 4.4 \\
\hline $95 \%$ confidence intervals & - & $2.3-2.9$ & $3.8-5.1$ \\
\hline \multicolumn{4}{|c|}{ Inclusion of only subjects taking investigated drug $\geq 90$ days $(n=42,4 \mid 3)$} \\
\hline Person-days of follow-up & $5,284,021$ & $2,658,961$ & 432,305 \\
\hline Number of ADR events & 509 & 676 & 214 \\
\hline ADR rate per 10,000 person-days & 1.0 & 2.5 & 5.0 \\
\hline Estimated rate ratio & I & 1.9 & 4.0 \\
\hline $95 \%$ confidence intervals & - & $1.7-2.2$ & $3.4-4.7$ \\
\hline \multicolumn{4}{|c|}{ Exclusion of coughing or cough as ADR events } \\
\hline Person-days of follow-up & $6,074,669$ & $2,997,958$ & 496,023 \\
\hline Number of ADR events & 859 & 970 & 325 \\
\hline ADR rate per 10,000 person-days & 1.4 & 3.2 & 6.6 \\
\hline Estimated rate ratio & I & 2.7 & 5.5 \\
\hline $95 \%$ confidence intervals & - & $2.5-3.0$ & $4.7-6.3$ \\
\hline \multicolumn{4}{|c|}{ Patient reclassification based on maximum number of medications used } \\
\hline Person-days of follow-up & $5,040,282$ & $3,119,006$ & $\mathrm{I}, 409,362$ \\
\hline Number of ADR events & 890 & 1543 & 711 \\
\hline ADR rate per 10,000 person-days & 1.8 & 4.9 & 5.0 \\
\hline Estimated rate ratio & I & 2.4 & 2.9 \\
\hline $95 \%$ confidence intervals & - & $2.2-2.6$ & $2.6-3.2$ \\
\hline
\end{tabular}

Note: ADR rate ratios were estimated using a Poisson regression model adjusted for age, sex, and drug category.

Abbreviation: ADR, adverse drug reaction.

the three groups according to the extent of concomitant drug use (results are shown in Table 2). To consider the discrepancy between drug use and actual risk period, sensitivity analysis was conducted after classifying the subjects according to the maximum number of medications taken at any point during the study period rather than the number being taken at initiation of the study period, thereby ensuring that the results were not biased due to misclassification of patients. The result of the analysis confirmed the existence of a positive association between ADR risk and number of co-medications taken.

Due to safety concerns, current clinical guidelines for the treatment of patients with uncontrolled hypertension recommend administration of combination therapies rather than increased doses of one medication. ${ }^{15,16}$ The results of this study, however, indicated that administration of multiple medications to elderly hypertensive patients is associated with increased risk of ADR, calling for increased care in the treatment of those patients by this practice. For those patients for whom administration of multiple medications was indicated, several literature reviews have suggested strategies, ${ }^{2,9,10,37,38}$ such as conducting medication reviews to identify unnecessary medications including over-thecounter drugs, ${ }^{39}$ the development of medication grids that display all medications and administration methods used by each patient in the form of a 7-day schedule to simplify medication regimens; ${ }^{40}$ the use of Beers criteria to identify inappropriate prescribing practices; ${ }^{41}$ and the development of interdisciplinary teams to perform regular assessments of medication regimens. ${ }^{42}$ These efforts can assist in determining the priority of medications used in a current regimen and promote rational use of medications.

The formation of interdisciplinary teams in which health professionals can share patient data to evaluate patient conditions as a whole and select minimally required pharmacotherapies has recently been recommended in Japan. ${ }^{43}$ This recommendation reflects recognition that the active contribution of community pharmacists may promote rational use of medications and reduce drug-related problems among the elderly, especially in aging societies. ${ }^{44}$ To ensure that elderly patients understand the reasons for and the correct ways of taking medications, and thereby decrease the risk of therapeutic failure as well as ADR, the role of pharmacists should be expanded to the education of patients and the monitoring of appropriate medication use, particularly in cases of polypharmacy. Comprehensive patient management with regular medication review should be conducted 
to determine the optimal number of medications and reduce the ADR risk for each patient.

\section{Limitations}

Although several sensitivity analyses were performed to consider potential biases, this study had certain limitations that may limit the generalizability of the findings. First, even though PMS studies must be conducted for all drugs prior to re-examination in Japan, the data regarding the results of only 21 PMS studies were contained in the database, as submission of these data was voluntary. Moreover, these data are relatively old, as they were collected from PMS studies conducted between 1981 and 1999, and were collected from few subjects who had taken diuretics and no subjects who had taken angiotensin-receptor blockers (ARBs) or combination drugs, the latter of which are now commonly used in the treatment of hypertensive patients. The RAD-AR Council recently developed a new database that includes the results of five studies of anti-hyperlipidemia drugs and, as of this writing, is preparing to add the results of three more antihypertensive drugs. ${ }^{45}$ Further research using data contained in the updated database is thus called for in the future.

Second, the study used PMS data that may itself have limitations due to misclassification of drug use status. Doctors often collect patient data using unreliable and subjective methods, such as by asking patients whether they have taken all of their medications without objectively confirming whether they have truly done so, leading to misclassification and possibly underestimation or overestimation of the risk of ADR. Third, the extent of patient adherence to treatment was not considered, which, particularly in the case of patients who might not have taken medications as prescribed, may have led to the underestimation of the risk of ADR.

\section{Conclusion}

Polypharmacy increases the risk of ADR in elderly Japanese patients with hypertension, calling for comprehensive management by regular medication review for determining the optimal number of medications to be prescribed to this patient population. Further studies are required to reduce the risk of drug-related problems in elderly Japanese patients.

\section{Acknowledgments}

Both authors contributed to the framework and theoretical design and were involved in the decision to submit the manuscript for publication. Izumi Sato performed the statistical analyses and drafted the manuscript, and Manabu Akazawa collected the data and provided inputs into the critical revisions. The authors thank the RAD-AR Council of Japan for allowing them to access the post-marketing survey data regarding the antihypertensive drugs examined in this study.

\section{Disclosure}

The authors received no financial support from industry or university grants to conduct this study.

\section{References}

1. World Health Organization Media Centre [homepage on the Internet] Medicines: rational use of medicines [Fact sheet $N^{\circ} 338$, May 2010]. Geneva: World Health Organization; 2010. Available from: http://www. who.int/mediacentre/factsheets/fs338/en/index.html. Accessed April 27, 2013.

2. Fulton MM, Allen ER. Polypharmacy in the elderly: a literature review. J Am Acad Nurse Pract. 2005;17(4):123-132.

3. Hilmer SN, Gnjidic D. The effects of polypharmacy in older adults. Clin Pharmacol Ther. 2008;85(1):86-88.

4. Aparasu RR, Mort JR, Brandt H. Polypharmacy trends in office visits by the elderly in the United States, 1990 and 2000. Res Social Adm Pharm. 2005;1(3):446-459.

5. Lee RD. Polypharmacy: a case report and new protocol for management. J Am Board Fam Pract. 1998;11(2):140-144.

6. Veehof LJ, Stewart RE, Meyboom-de Jong B, Haaijer-Ruskamp FM. Adverse drug reactions and polypharmacy in the elderly in general practice. Eur J Clin Pharmacol. 1999;55(7):533-536.

7. Nguyen JK, Fouts MM, Kotabe SE, Lo E. Polypharmacy as a risk factor for adverse drug reactions in geriatric nursing home residents. $A m$ J Geriatr Pharmacother. 2006;4(1):36-41.

8. Gallagher P, Barry P, O'Mahony D. Inappropriate prescribing in the elderly. J Clin Pharm Ther. 2007;32(2):113-121.

9. Hajjar ER, Cafiero AC, Hanlon JT. Polypharmacy in elderly patients. Am J Geriatr Pharmacother. 2007;5(4):345-351.

10. Salazar JA, Poon I, Nair M. Clinical consequences of polypharmacy in elderly: expect the unexpected, think the unthinkable. Expert Opin Drug Saf. 2007;6(6):695-704.

11. Ministry of Health, Labour and Welfare. [homepage on the Internet, in Japanese]. Patient Survey 2008. Tokyo: Ministry of Health, Labour and Welfare; 2008 [cited December 3, 2009]. Available from: http:// www.mhlw.go.jp/toukei/saikin/hw/kanja/08/index.html. Accessed April 27, 2013.

12. Suzuki Y, Akishita M, Arai H, Teramoto S, Morimoto S, Toba K. Multiple consultations and polypharmacy of patients attending geriatric outpatient units of university hospitals. Geriatr Gerontol Int. 2006;6(4):244-247.

13. Bangalore S, Kamalakkannan G, Parkar S, Messerli FH. Fixed-dose combinations improve medication compliance: a meta-analysis. Am J Med. 2007;120(8):713-719.

14. Reid JL. Fall and rise of polypharmacy. Hypertension. 2007;49(2): 266-267.

15. Frank J. Managing hypertension using combination therapy. Am Fam Physician. 2008;77(9):1279-1286.

16. Matsuoka H. Large clinical trials of ARB in Japan and its reflection to new guidelines. Nihon Rinsho. 2009;67(4):669-674. Japanese.

17. Koshy S, Bakris GL. Therapeutic approaches to achieve desired blood pressure goals: focus on calcium channel blockers. Cardiovasc Drugs Ther. 2000;14(3):295-301.

18. Steinman MA, Landefeld CS, Rosenthal GE, Berthenthal D, Sen S, Kaboli PJ. Polypharmacy and prescribing quality in older people. $J \mathrm{Am}$ Geriatr Soc. 2006;54(10):1516-1523.

19. Cooney D, Pascuzzi K. Polypharmacy in the elderly: focus on drug interactions and adherence in hypertension. Clin Geriatr Med. 2009;25(2): 221-233. 
20. Arai H, Akihita M, Teramoto S, et al. Incidence of adverse drug reactions in geriatric units of university hospitals. Geriatr Gerntlo Int. 2005;5(4):293-297.

21. Akishita M, Toba K, Nagano K, Ouchi Y. Adverse drug reactions in older people with dementia. J Am Geriatr Soc. 2002;50(2):400-401.

22. Tanaka K, Morita Y, Kawabe E, Kubota K. Drug use investigation (DUI) and prescription-event monitoring in Japan (J-PEM). Pharmacoepidemiol Drug Saf. 2001;10(7):653-658.

23. Kubota K, Koyama H. Pharmacovigilance and Risk Management in Japan. In: Mann RD, Andrews EB, editors. Pharmacovigilance. 2nd ed. Chichester, UK: John Wiley and Sons, Ltd; 2007:387-394.

24. Matsushita Y, Sugihara M, Kawasugi K. Integration of Drug UseResults Survey (DUS) Data of a Pharmaceutical Manufacturer. Jpn J Pharmacoepidemiol. 2010;15(2):61-71.

25. Risk/Benefit Assessment of Drugs - Analysis and Response Council. Database for Drug Use-Results Survey, Antihypertensives/Antibacterial agents. Available from: http://www.rad-ar.or.jp/01/05_datebase/ kitei+form/DB_DURS_leaflet.pdf. Accessed April 27, 2013.

26. Fujita T, Miura Y, Mayama T. A pilot study to build a database on seven anti-hypertensive drugs. Pharmacoepidemiol Drug Saf. 2005;14(1):41-46.

27. Centers for Disease Control and Prevention [webpage on the Internet]. International Classification of Diseases, Ninth Revision, Clinical Modification (ICD-9-CM). Atlanta: Centers for Disease Control and Prevention [updated October 4, 2012]. Available from: http://www. cdc.gov/nchs/icd/icd9 cm.htm. Accessed April 27, 2013.

28. Medical Dictionary for Regulatory Activities Maintenance and Support Services Organization [homepage on the Internet]. MedDRA - the Medical Dictionary for Regulatory Activities [updated March 1, 2013]. Available from: http://www.meddramsso.com/index.asp. Accessed April 27, 2013.

29. Yokotsuka M, Aoyama M, Kubota K. The use of a medical dictionary for regulatory activities terminology (MedDRA) in prescriptionevent monitoring in Japan (J-PEM). Int J Med Inform. 2000;57(2-3): 139-153.

30. Ministry of Health, Labour and Welfare. Drug Classification Codes for Internal Medicines. Tokyo: Ministry of Health, Labour and Welfare; 2013 [updated April 16, 2013]. Available from: http://www.mhlw.go.jp/ topics/2012/03/dl/tp130418-01_1.pdf. Accessed April 27, 2013.

31. Fujita T, Mayama T. [A database of anti-hypertensive drugs from Drug Use Investigations and its practical use example]. Japan Statistical Society. 2007;36(2):205-217. Japanese [with English abstract].

32. Yoshida M, Matsumoto T, Suzuki T, Kitamura S, Mayama T. Effect of concomitant treatment with a CYP3A4 inhibitor and a calcium channel blocker. Pharmacoepidemiol Drug Saf. 2008;17(1):70-75.

33. Ishiguro C, Fujita T, Omori T, Fujii Y, Mayama T, Sato T. Assessing the effects of non-steroidal anti-inflammatory drugs on antihypertensive drug therapy using post-marketing surveillance database. J Epidemiol. 2008;18(3):119-124.
34. Risk/Benefit Assessment of Drugs - Analysis and Response Council. PE/SOP2 Administrative Rules and Regulations of Database for Drug Use-Result Surveys [cited June 2011]. Available from: http://www. rad-ar.or.jp/01/05_datebase/kitei+form/PE_DB_kanri20110628en.pdf. Accessed April 27, 2013.

35. Ray WA, Chung CP, Murray KT, Hall K, Stein CM. Atypical antipsychotic drugs and the risk of sudden cardiac death. $N$ Engl J Med. 2009;360(3):225-235.

36. McAfee AT, Ming EE, Seeger JD, et al. The comparative safety of rosuvastatin: a retrospective matched cohort study in over 48,000 initiators of statin therapy. Pharmacoepidemiol Drug Saf. 2006;15(7):444-453.

37. Rollason V, Vogt N. Reduction of polypharmacy in the elderly: a systematic review of the role of the pharmacist. Drugs Aging. 2003;20(11):817-832.

38. Maeda K. Systematic review of the effects of improvement of prescription to reduce the number of medications in the elderly with Polypharmacy. Yakugaku Zasshi. 2009;129(5):631-645.

39. Fillit HM, Futterman R, Orland BI, et al. Polypharmacy management in Medicare managed care: changes in prescribing by primary care physicians resulting from a program promoting medication reviews. Am J Manag Care. 1999;5(5):587-594.

40. Muir AJ, Sanders LL, Wilkinson WE, Schmader K. Reducing medication regimen complexity: a controlled trial. J Gen Intern Med. 2001;16(2):77-82.

41. Fick DM, Maclean JR, Rodriguez NA, et al. A randomized study to decrease the use of potentially inappropriate medications among community-dwelling older adults in a southeastern managed care organization. Am J Manag Care. 2004;10(11 Pt 1):761-768.

42. Schmader KE, Hanlon JT, Pieper CF, et al. Effects of geriatric evaluation and management on adverse drug reactions and suboptimal prescribing in the frail elderly. Am J Med. 2004;116(6):394-401.

43. Ministry of Health, Labour and Welfare. Health Policy Publication 0430-1. Tokyo: Ministry of Health, Labour and Welfare; 2010 [cited April 30, 2010]. Available from: http://wwwhourei.mhlw.go.jp/hourei/ doc/tsuchi/T100506G0010.pdf. Accessed April 27, 2013.

44. Yamamura S, Yamamoto N, Oide S, Kitazawa S. Current state of community pharmacy in Japan: practice, research, and future opportunities or challenges. Ann Pharmacother. 2006;40(11):2008-2014.

45. Watanabe S, Nakano Y, Nomura K. [Steps in developing a database of drug use-result surveillance: as an example of anti-hyperlipidemia drugs]. Jpn J Pharmacoepidemiol. 2012;17(2):87-97. Japanese [with English abstract].
Drug, Healthcare and Patient Safety

\section{Publish your work in this journal}

Drug, Healthcare and Patient Safety is an international, peer-reviewed open-access journal exploring patient safety issues in the healthcare continuum from diagnostic and screening interventions through to treatment, drug therapy and surgery. The journal is characterized by the rapid reporting of reviews, original research, clinical, epidemiological and

\section{Dovepress}

post-marketing surveillance studies, risk management, health literacy and educational programs across all areas of healthcare delivery. The manuscript management system is completely online and includes a very quick and fair peer-review system. Visit http://www.dovepress.com/ testimonials.php to read real quotes from published authors. 